\title{
LA REACCION DE VOLLMER EN EL DIAGNOSTICO DE LA TUBERCULOSIS INFANTIL
}

\author{
Por los Dires. \\ JORGE PEÑA CERECEDA \\ Tíbiogo del Ho pital Manu Al Arriarán. \\ inédro-Jife del Sanatorio "Los Guindos" \\ JULIO MENEGHELLO RIVERA \\ Médico re:idente del Sanatorio "Los Guindos". \\ Ayudante de la Catedra de Battcriología dei Profesot Hugo Bateroro, \\ Médico del Servicio de Infeccioses del Hospital Manucl Arriarán.
}

\author{
LUIS MORALES FUENTES \\ Ayudanti ad-honotem del Hospital Artiarán.
}

Reacción de Vollmer.

Desde la aplicación de la tuberculina al diagnóstico ae la tuberculosis, especialménte en el niño, realizada por von Pirquet en 1907, se han ensayado numerosos procedimientos que tenden, unos a obtener una mayor sensibilidad a la tuberculina y otros a simplificat la técnica de las reacciones. Entre las primeras citatemos la prueba de Mantoux y entre las segundas. las diferentes reacciones percutáneas introducirlas por Moro.

No nos detendremos a estudiar en detalle cada ntno de los innumerables métodos empleados para poner en evidencra la sensibilidad a la tuberculina en un organismo tuberctizado; nos limitaremos solamente a estadiar, en forma comparativa, las ventajas y los inconvenientes que presentan en 13 clínica los más usados de estos procedimientos.

En la práctica diaria, especialmente cuando se hace la investigación tuberculínica en colectividades como Escuelas, A iss, etc., basta con la reacción de Pirquet que, si bien es $f \quad: 0, \mathrm{da}$ un margen apreciable de errores, tiene a su favor 
la facilidad de su técrica. Pero cuando se desea hacer esia investigación con más acuciosidad o en casos de diagnósticos difíciles, es necesario recurrir a las pruebas intradérmicas. Fste procedimiento tiene la ventaja de permitir un aumento de !as concentraciones de tuberculina y de evitar posibles reacciones tocales o generales. Puede decirse que en la actualidad son éstos los dos únicos procedimientos empleados por la mayoria de los pediatras para èl diagnóstico de la tuberculosis infantil; aunque algunos prefieren comenzar directamente por concentraciones débiles de tuberculina por vía intradérmica. prescindiendo del Pirquet. En todo caso. cualquiera que sea el método empleado, las cantidades de tuberculina inyectada son sensiblemente las mismas, porque una reacción de Pirquer cotresponde, aproximadamente, a un Mantoux al 1 x 10.000 .

El método percutáneo fué introducido por Moro, el que empleó una preparación otiginal a base de lanolina y tuberculina en partes iguales. agregando sustancias queratoliticas para facilitar su absorción.

El autor elmpleaba el procedimiento de la frotación en un pequeño espacio de piel en la región sub-clavicular con la pomada ya indicada, obteniendo, al cabo de 48 horas, una erupción pápulo-eritematosa. más o menos intensa, según los casos. El método percutáneo ha sido abandonado, porque sus resultados son inconstantes, ya que dependen. en gran parte, de factores personales muy difíciles de evitar. Por 10 demás. la sensibilídad de este método es muy inferior al de Pirquet.

Reacción de Vollmer. - Esta prueba tuberculínica. ideada por Vollmer en 1937, ha sido ensayada por diferentes autores, especialmente norteamericanos y los resultados obtenidos concuerdan en el sentido de que su sensibilidad es mayor que la de Mantoux al 1 x 1.000. Por otra parte, las ventajas de su técnica más sencilla hacen que su empleo se haya difundido rápidamente en los círculos pediátricos.

Técnica, - Describiremos la técnica empleada por el autor y en seguida sus diversas modificaciones, que no alteran substancialmente el procedimiento primitivo.

Se cortan tres cuadrados de papel de filtro común de $1 \mathrm{~cm}$. por lado. de los cuales dos se imbiben con tuberculina bruta de Koch y el tercero, que sirve de control, con suero fenicado. Los dos trozos de papel con tuberculina se pegan en los extremos de un pedazo de tela adhesiva de $2,5 \mathrm{cms}$, de 
ancho por $7,5 \mathrm{cms}$. de largo y en el centro se coloca el tercer cuadradito de papel embebido con suero fenicado. Este parche se adhiere a la piel de la región esternal, dorsal o del antebrazo, previa limpieza con éter, acetona o bencina. Se han escogido estas regiones por estar desprovistas de pelos y por ser más accesibles al control.

El parche se saca después de 48 horas y los resultados de la reacción pueden leerse inmediatamente o en las 48 horas siguientes. Por lo común, este último plazo es más recomendable, ya que suelen aparecer reacciones tardías. La lectura de la reacción de Vollmer es muy sencilla y para comprender mejor sus diversas modalidades, su autor distingue los siguientes 4 grados fundamentales:

1. Pequeñas eflorescencias de tipo liquenoídeo.

2. Erupciones liquenoídeo-foliculares confluentes que se limitan al área del cuadradito de papel.

3. Erupciones confluentes con marcada infiltración altededor del cuadrado.

4. La reacción se extiende más allá de la superficie del cuadrado con formación de flictenas.

Cuando la erupción es muy intensa, se observa prurito, por lo que muchos niños se sacan la tela antes de las 48 horas (por eso es más recomendable la región dorsal, sobre todo en los niños pequeños).

La duración de la reacción es más n menos de una semana, observándose una decoloración paulatina y luego después una pigmentación semejante a la que se ve en las reacciones de Pirquet o Mantoux después de algunos días.

La tuberculina que se emplea en la reacción de Vollmer es, por lo común, la T. V. K. (tuberculina vieja de Koch). pero el autor ha utilizado tambien la tuberculina concentrada purificada (P. P. D.).

Una de las modificaciones de la reacción de Vollmer la constituye la ideada por "The danisch State Serum Institute" de Copenhague. Esta institución emplea rina mezcla de tuberculina antigua de Koch y P. P. D. en proporción de 3 a 1 , conservándola activa por espacio de un año en una atmósfera fría. Una cantidad suficiente de esta mezcla para cubrir una moneda de tres peniques se aprieta con un pedazo de tela adhesiva y se aplica a la piel por debajo de la clavícula, después de limpiarla con acetona. El parche se retira a las 48 horas. Como control utilizan un simiple pedazo de tela aplica- 
do al lado opuesto. La institución mencionada asegura que esta mezcla de tuberculina actúa con mayor rapidez (24 hotas;.

El parche se emplea comunimente con tuberculina fresca: pero puede conservarse durante un año, según Vollmet, sin que se altere la acción de la tuberculina. Para esto el autor recomienda cubrirlo con us trozo de linón.

La desecación no es un inconveniente para la absorción de la tuberculina por la piel, porque se humedece por la perspiración, más rápidamente en las épocas de calor que en las frías, factor que es preciso tomar en cuenta al efectuar el control de la reacción. En efecto; durante la estación fría. los resultados pueden aparecer tardiamente, mientras que en Verano éstos son mucho más precoces.

Hay que hacer notar que nunca se han observado reacciones focales o generales con el método de Vollmer, a pesłr de emplearse tuberculina pura o soluciones muy concentra. das de P. P. D. A estas mismas conclusiones llegan todo: los autores que se han preocupado de este tema. orincipalmente los americainos.

Fstudio clínico de la reacción de Volimer. - Desde 1937 a mayo de 1940 , fecha de la última publicación de Vollmer, el valor del Patch test ha sido estudiado en 2.000 niños divididos en tres grupos:

Grupo I. - Se trata de 847 niños, del Mount Sinai Hospital que fueron sometidos a examen clinico. roentgeno. gráfico y a las pruebas tuberculínicas del Mantoux y Patch test. Entre estos niños hubo uno sólo en que; à pesar de acusar una infección tuberculosa franca, faltaba la reacción positiva al parche. Se trataba de una tuberculosis miliar, eit la que también la prueba de Mantoux al $1 \%$ fué negativa.

Jas reacciones de Mantoux y del Patcb test se hicieron en 666 niñoi y dieron 657 resultados positivos para ambas reacciones y sólo en 9 casos hubo disconformidad.

Grupo II. - Comprende 540 niños del Sea View Hospiral, Staten Island, N. Y., con tuberculosis activa. Se prac.. ticó la reacción de Vollmer en 535 casos, obteniéndose 529 resuitados positives y 6 negativos. Estos últimos dieron también reacción negativa al Mantoux a concentraciones altas de tuberculina, en varias ocasiones.

Grupo III. - En la primavera de 1938, 613 estudiantes del Stuyvesant High School N. Y., fueron controlados 
por el Departamento de Salubridad de Nueva York, con Is prueba de Mantoux, exámenes de Rayos X y Patch test. Los médicos que controlaron esta reacción leyeton los resultados a las 24 horas en vez de 48 . como es lo correcto, lo que hizo variar los porcentajes. Sin embargo, 461 reacciones fueron negativas a ambas reacciones, 125 positivas. 18 negativas al Patch test $y$ positivas al Mantoux, 4 positivas al Vollmer y negativas al Mantoux y 5 dudosas al Vollmer y negativas al Mantoux. Esta última reacción se hizo a una concentración del $1 \%$.

Otros autores, entre ellos Taylor, Steward, Court, Saul y Greenwald han comparado también la reacción de Vollmer con el Mantoux, usando T. V. K. al $1 \%$ exclusivamente Greenwald encuentra un $100 \%$ de acuerdo, Taylor, Steward y Saul encuentran superior el Mantoux al parche y sólo Court lo considera levemente inferior. Por su parte, Weiner y Neustadt han becho estudios comparativos entre el Pirquet y el método de Vollmer en 257 niños indigentes. negros en un $90 \%$. En los casos negativos al Pirquet se aplicó el Mantoux en dosis de $0,1 \mathrm{cc}$. de tuna solución de T. V. K. al $1 \%$. En 200 casos, el parche y el Pirquet fueron negativos. En 44 casos la reacción fué positiva y en 13 casos hubo desacuerdo entre el Patch test y el Pirquet. Se practicó la reacción de Mantoux en 63 niños en los que el Patch test $y$ el Pirquet habían sido negativos. De éstos, 60 dieron reacción negativa al Mantoux y 3 positiva al mismo.

Weiner y Neustadt llegan a la conclusión de que el Patch test es tan satisfactorio como el Pirquet.

Hart y Shaw comparan la reacción de Vollmer con el Mantonx: pero aumentan las concentraciones $y$ usan la $\mathrm{T}$. $V$. K. al $\mathrm{I}$ \% en aquellos casos que han reaccionado negativamente a soluciones más diluídas.

Como puede observarse, la proporción de casos favorabJes a la reacción de Vollmer encontrado por los autores ci. tados coincide, aproximadamente, con la obtenida por el autor. Tomando la totalidad de los casos de Vollmer y demás invedrigadorcs, se obtienen. 6,104 casos con 1,819 reacciones positivas al $P_{\text {atch }}$ test comparados con 1.833 positivos al Mantoux, incluyendo el uso de concentraciones más altas de tuberculina ( $1 \%$ de T. V. K. y P. P. D. en dos dosis). La diferencia es sólo de $0,77 \%$. Si. las reacciones poritivas al Vollmer ce compara sólo con las positiviss al Manconx, usando concencraciones no más altas del $1 \%$ de 
T. V. K. o P. P. D. en la dosịg débil da un $100 \%$ de equávalencia.

Peck y Wegman, en completo desacueido con los resultados anteriores, encuentran 28,3 y $70,5 \%$ de discrepancia respectivamente, entre el método de Vollmer y el Mantoux con P. P. D. en $1 .^{a}$ y $2 .^{\text {a }}$ dosis.

Vollmer atribuye esto a diversas causas: en primet lugar a errores de dilación de la tuberculina. a contaminaciones de la solución, a mala interpretación de la reacción, a parches yiejos cuya tela ha perdido su poder adhesivo, a que là piel no ha quedado lo suficientemente limpiar o a que el parche ha sido removido antes de las 48 horas. Con el fin de evitar estos inconvenientes. Vollmer sugiere una pauta para la correcta realización de la prueba: retirado.

1. El parche debe controlarse dos días después de ser

2. Las reacciones negativas deben ser comparadas con el Mantoux al $1 \%$, o bien, con la segunda dosis de P. P. D.

3. Ambos test deben repetirse si hay alguna discrepancia, ya que suelen las concentraciones altas de tuberculina, ocasionar pseudo-reacciones.

4. Sí la discrepancia continúa, la reacción de Mantoux sé considerará como la decisiva.

Nuestras experiencias. - Por nuestra parte nos hemos preocupado de estudiar esta reacción con el propósito de apreciar su sensibilidad frente a las pruebas tuberculínicas comunmente enipleadas en nuestro medio, al mismo tiempo que corroborar las investigaciones practicadas por autores extranjeros. En líneas generales, podemos decir que los resultados obtenidos en más de 1,000 casos, concuerdan aproximadamente con los de Vollmer en los EE. UU., como veremos más adelante.

En cuanto a la técnica de la reacción de Vollmer, en un comienzo seguimos exactamente la recomendada: por su antor; pero después la hemos simplificado, dejando en la tela sólo nno de los papeles impregnados con tuberculina y en el otro el control con suero fenicado.

Este procedimiento nos ha resultado igual al empleais primitivamente, significando una buena economía $y$ una mayor sencillez en la técnica.

Otro detalle que creemos de interés consignar es el relarivo a la manera de preparar los parches en serie con el má- 
rimum de economía en tuberculina. Para esto hemos preparado una banda de papel de filtro de $1 \mathrm{~cm}$. de ancho por 60 cms. de largo, la que se impregna de tuberculina, haciéndola pasar lentamente sobre la superficie de un plato o de una plancha de vidrio, en la que se han colocado 2 a $3 \mathrm{cc}$. de tuberculina. Este procedimiento permite hacer la impregalación de la banda de papel en forma homogénea, empleando la cantidad de tuberculina estrictamente necesaria. En séguida se corta el papel en trozos de $1 \mathrm{~cm}$. Este método tiene la ventaja, además, de poder conservar las tiras de papel con tuberculina durante un largo tiempo (3 meses); aunque, como lo hemos podido observar, la reacción de Vollmer es mucho más segura y más intensa cuando se pracrica con tuberculina fresca. Otra modificación consiste en cubrir el parche ya preparado, no con un trozo de linón. como lo bace Vollmer, sino con un pedazo de tela de dibujante, lo que permite mantener la actividad de la ruberculina por un tiempo mayor. De esta manera hemos podido obtener reacciones positivas con parches preparados 6 meses antes.

En algunos casos de negatividad de la reacción de Vollmer, la hamos repetido con el trozo de papel impregnado dos veces en tuberculina. Este sistema puede compararse, aproximadamente, al empleado por el Instituto de Copenhague con P. P. D.

Otra modificación en la técnica, fácil de realizar y que es recomendable en los casos dudosos, consiste en repetir la prueba de Vollmer en el mismo sitio de la anterior, antes de 24 horas. Por último, queremos insistir en un detalle que tiene cierta importancia en algunos casos y que consiste en utilizar, para la limpieza de la piel, una sustancia que se evapore rápidamente como el éter o la bencina, porque si la piel permanece húmeda en el momento de aplicarse el parche, éste se desprende pocas horas después, con el fracaso consiguiente de la reacción. Durante nuestras investigaciones nos ha sido posible observar:

1. Que algunas reacciones son positivas en períodos muy cortos ( 20 boras), como pudimos observar en varios niños que se sacaron el parche antes del tiempo indicado.

2. En los exudativos hemos visto verdaderas ulceraciones de la piel con la reacción de Vollmer, por lo que habría que agregar un quinto tipo a los descritos por el autor. 
3. Que en algunos niños cuya piol es muy fina, se observa un eritema que abarca toda la extensión de la tela: pero este eritema es fugaz y contrasta con la coloración rojo oscura, más o menos infilirada, que da la reacción de Vollmer.

Método empleado en nuestro estudio de la reacción de Vollmer. - Dividiremos nuestras investigaciones en dos partes: una que comprende un grupo de niños tomados al azar, sin diagnóstico de tuberculosis y otro que abarca un grupo de niños catalogados como tuberculosos.

Primera parte. - Comprende tres sub-grupos:

Primer sub-grupo: Se refiere a una investigación con pruebas de Pirquet y Mantoux en 660 niños tomados en "Centros de Defensa" y en Policlínicos generales, en edades que oscilan entre 1 y 12 años, sin estudio previo tuberculínico ni radiológico:

\begin{tabular}{ccc} 
Casos & Pirquet & Vollmer \\
\hline 329 & + & + \\
272 & - & + \\
46 & + & + \\
19 & $-5 \%$ & $\frac{+}{55.9 \%}$
\end{tabular}

Como vernos, existe una proporción del $3,9 \%$ de mayor positividad del Vollmer en relación con el Pirquet.

Segundo sub-grupo: Se trata de 28 niños tomados de entre los que presentaton el Vollmer y el Pirquet negativos en el grupo anterior. En ellos se practicó el Man,toux á $I \times 1,000$ junto con la prueba de Vollmer por segunda vez. dos meses después de efectuada las primeras reacciones. Resultaron positivos. tanto al Vollmet como al Mantoux. 4 casos: 3 lo fueron sólo al Mantoux y 2 al Vollmer. El porcentaje de reacciones positivas al Vollmer es de $21 \%$ y al Mantoux de $25 \%$. Como vemos. los resultados son ligera. minte favorables a la prueba de Mantoux.

Tercer sub-grupo: Hemos hecho también. un estudio de las pruebas de Mantoux al 1 x 1,000 y de Vollmer en 354 
casos, sin practicar previamente el Pirquet. En el cuadro siguiente, damos los resultados:

\begin{tabular}{rcc}
\hline Cisos & Mantoux $1 \times 1.000$ & Volluse \\
\hline $\begin{aligned} 209 \\
106\end{aligned}$ & + & + \\
31 & - & 1 \\
8 & $\frac{+}{61 \%}$ & $\frac{-}{67.7 \%}$
\end{tabular}

Fl estudio de las cifras que arroja este cuadro es muy interesante, porque la mayor positividad de la reacción di Vollmer sobre la de Mantoux alcanza a un $6,7 \%$. la cifra más alta que se ha alcanzado hasta ahora. Este resultado nos permite establecer que, en una investigación de la sensibilidad tuberculínica en niños no catalogados como tuberculosos, puede reemplazarse con ventajas la reacción de Mantoux por la de Vollmer.

Con el objeto de agotar la investigación, se repitió la prueba de Vollmer, comparándola con la reacción de Man. toux al $1 \times 1,000$, en un grupo de 30 niños tomados al azat de entre los que dieron reacciones negativas en el grupo anterior. Los resultados fueron los siguientes: 4 casos fueron positjvos al Mantoux exclusivamente y 3 positivos al Vollmer $y$ al Mantoux. El resto, o sea, 23 niños. continuaron con sus reacciones negativas.

$A$ un grupo de 12 niños con Pirquet negativo y Vollnter positivo, se le hizo la prueba de Mantoux al 1 x 1,000 y de Vollmer nuevamente, con los resultados siguientes:

5 casos reaccionaron positivamente al Mantoux y en la totalidad de ellos se obtuvo nuevamente la positividad del Vollmer.

Segunda parte. - Se refiere a 216 niños investigados ell el Sanatorio para niños tuberculosos de "Los Guindos" $Y$ del Servicio de Tisiología del Hospital "Manuel Arriarán".

\begin{tabular}{|c|c|c|c|}
\hline & Casos & Pirquet & Vollmics \\
\hline & 169 & $-i$ & $\dot{+}$ \\
\hline & 22 & 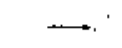 & - \\
\hline & 16 & $\therefore-$ & 1 \\
\hline . & 9 & + & $\rightarrow$ \\
\hline Total & 216 & $82 \%$ & $85,6 \%$ \\
\hline
\end{tabular}


En este grupo se puede apreciar también una proporción de $3.6 \%$ de mayor positividad de la reacción de Vollmer. Al mismo tiempo se hizo la investigación, en este grupo, del Mantoux al 1 x 1,000 en 20 casos de los que tesultaron negativos al Pirquet y al Vollmer, como puede apreciarse en el cuadrc siguiente:

\begin{tabular}{rccc} 
Casos & Mantorx $1 \times 1,000$ & Vollmer \\
\hline 16 & + & + \\
1 & - & - \\
2 & + & - \\
1 & -1 & $90 \%$
\end{tabular}

Como vemos, el resultado es ampliamente favorable al Vollmer con una mayor positividad de un $5 \%$.

Como ula a observación de interés presentamos el caso de una niña, en la cual sólo se pudo evidenciar la sensibilidad tuberculínica por medio de la reacción de Vollmer después de haber dado resultados negativos el Mantoux al 1 x 1,000, repetidos en dos ocasiones.

Obs. N. 1,282.-Hospital Arriarán, 12 años.

Antecedentes: 6 hermanos fallecidos de meningitis. Coqueluche y sarampión a los 8 años.

Enfermedad actual: Ingresa al servicio de Medicina el 15 de abril de 1940 por un cuadro de asistolía. Como presentara signos de rondensación pulmonar, se le practica un Mantoux al 1 x 1,000, que resulta negativo. Cuatro días después $s_{i}$ practica un Vollmer, que resulta débilmente positivo. ta negativo.

El Mantoux repetido nuevamente al $1 \times 1,000$, resul.

Fallece una semana después y la autopsia nos revela una pleuritis fibrosa del tercio inferior del lóbulo superior izquierdo y ganglios paratraqueales y de la bifurcación del tamaño de fréjoles que, al examen histológico, dan una fibrosis con zonas de calcificación. seguramente de origen tuberculoso.

Calidad de la taberculina. - Con el objeto de comprobar la sensibilidad y constancia de las diferentes tuberculinas empleadas entre nosotros, hemos hecho un estudio comparativo de laberculina preparada en nuestro Instituto Bac. 
teriológico con la de Bayer y con la P. P. D. argentina, que nos ha sido enviada gentilmente por nuestro amigo, Dr. Flocencio Escardó.

El procedimiento empleado ha sido el siguiente:

Para la reacción de Pirquet se ha practicado una escarificación con tuberculina Bayer en la parte alta del antebrazo y oura con tuberculina naciomial en la parte inferior. Respecto a la reacción de Vollmer, se ha procedido a impregnar uno de los cuadraditos de papel con tuberculina Bayer y el otro con tuberculina nacional, colocando el control en el centro. De esta manera hemos podido comparar, en una forma más - menos exacta, tanto la coloración como la infiltración de ambas tuberculinas.

Los resultados los exponemos en el cuadro siguiente:

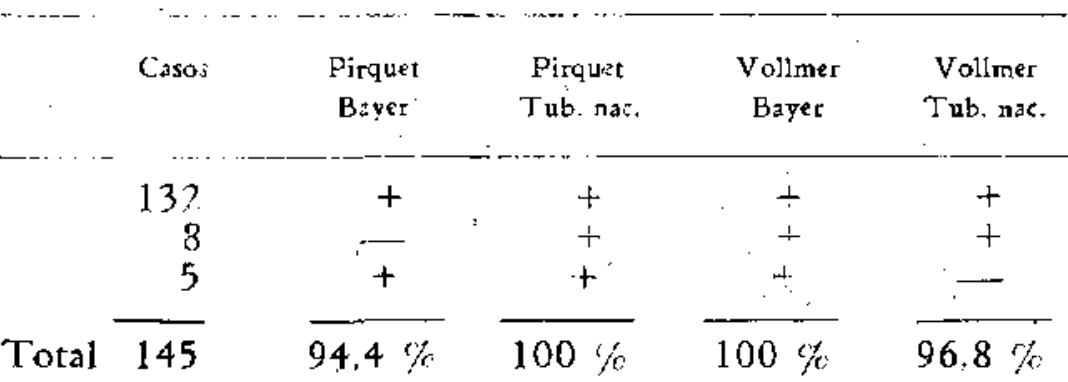

También hemos practicado algunas réacciones de Mantoux con ambas tuberculinas, siguiendo el mismo procediiniento empleado para el Pirquet. Los resultados obtenidos en 25 casos, coinciden casi exactamente, por lo que nos ahorramos todo comentario.

Por último, diremos algunas palabras sobre los resultados obtenidos en 50 casos, en los que se practicaron las pruebas de Vollmer y de Mantoux con la P. P. D. (proténa purificada derivativa) a la dosis de 0.0025 milígramo (dosis débil). De los 50 niños estudiados, 47 reaccionaron positivamente al Vollmer $(94 \%)$ y 45 à la P. P. D. $(90 \%)$. Existe, pues, una mayor positividad del Vollmer sobre la P. P. D. débil equivalente a un $4 \%$ \%

En un segundo grupo de 20 niños con tuberculinas negativas, tanto at Mantoux al $1 \times 1,000$ como a la P. P. D. débil, se practicó la pruebá de Vollmer con T. V.K. y la de 
Mantoux con P. P. D. fuerte (0.25 milígramo), Los resultados fueron los siguientes:

Los 20 niños reaccionaron positivamente a la P. P. D. fuerte con grandes reacciones y sólo 12 dieron resultados po. sitivos al Vollmer.

Finalmente, repetimos el Vollmer en un grupo que antexiormente había dado resultados negativos a esta reacción, con una mezcla en partes iguales, de T. V. K. y de P. P. D. fuerte (modificación de la técnica recomendada por el Instituto de Copenhague): De 10 niños sometidos a esta prueba. 9 reaccionaron positivamente en forma interisa y sólo uno dió resultado negativo. (Es de advertir que este caso dió resultados constantemente negativos al Mantoux a diferentes concentraciones y aun a la P. P. D. fuerte).

\section{Comentario general y condusiones.}

Las estadísticas practicadas, tanto por autores extranjeros como por nosotros, demuestran que la reacción de Vollmer tiene una sensibilidad ligeramente superior a la prueba de Mantoux al $1 \times 1,000$, siendo mucho más constante que la de Pirquet. Tiene la ventaja, sobre esta última, de ser más sencilla en su téenica y de no provocar traumatismos que. como es sabido, despiertan resistencias de parte de las familias de los enfermos. Por otra parte, tiene sobre el Mantoux, fuera de las ventajas ya anotadas, la de no provocar reacciones de ninguna especie, como lo confitma la unanimidad de los autores que se han preocupado de esta materia.

Las simplificaciones que hemos introducido en algunos detalles de la reacción de Vollmer, nos han significado tuna e:onomía bastante apreciable, sin que pudiera observarse ninguna variación en los resultados. Recomendamos que; en 10 posible, se emplee tuberculina fresca, aunque también. pueden obtenerse buenos resultados con la tuberculina desecada, pero no en forma tan constante. El procedimiento que empleamos en un buen número de casos y que coinsiste en impregnar dos veces el papel de filtro con tuberculina, es, a nuestro juicio, el más eficaz, porque evita el inconveniente de la excesiva desecación que suele observarse en la piel después de su limpieza con éter o con bencita. Débe hacerse la segunda impregnación en el momento mismo de realizar la reacción. El método recomendado por el Instituto de Copenhague de emplear 
una mezcla al $1 \times 3$ de P. P. D. fuerte y de T. V. K., nos parece excelente, a pesar de que nosotros hemos usado una mezcla al medio. Debe usarse, eso sí, una mezcla reciente para obtener buenos resultados. Tiene el inconveniente este método de ser más costoso por el empleo de la P. P. D., sustancia inestable que so puede conservarse durante un tietapo ccrto en solución y que aun ino se emplea en forma constante en la práctica diaria. En todo caso. nuestra experiencia es nuy escasa al respecto y el procedimiento vale la pena que se ensave en forma más amplia.

Los cuatro tipos de reacciones descritos por Vollmer se nbservan más o menos exactamente en la práctica; el quinto tipo, que hemos descrito, es excepcional y sólo se encuentra en los diatésicos o en niños con graves alteraciones de su estado nutritivo.

Estimamos que la reacción de Vollmer constituye un valioso aporte para la investigación de la elergía tuberculosa en los niños, tanto por su alto grado de sensibilidad como por la sencillez de su técnica.

\section{Conclusiones}

1. La reacción de Vollmer tiene un $3,9 \%$ de mayor sensibilidad que la de Pirquet y un $6,7 \%$ que la de Mantoux al $1 \times 1,000$.

2. Recomendamos la reacción de Vollmer para la in vestigación de la sensibilidad tuberculínica en las grandes colectividades por la sencillez de su técnica, y la de Mantoux para el estudio más exacto de la alergia, ya que puede emplearse a distintas concentraciones.

3. La mezcla de tuberculina vieja de Koch (T. V. K.) con P. P. D. fuerte, aumenta considerablemente la eficacia de la reacción de Vollmer.

4. El método de Vollmer no da reacciones focales ni generales.

5. Los distintos tipos de tuberculina vieja de Koch (Bayer, Instituto Bacteriológico de Chile, etc.) no bacen variar substancialmente los resultados de las reacciones tuberculínicas, sea cual fuere el método empleado. 


\section{BIBLIOGRAFIA}

VOLLMER and GOLDBERGER. - Tha Amitican Journil of Diseases af Childrin. Junio 1939. Vol. 57, N96.

HART. F. D. - Lancet 2: 609. Sip. 1938.

VOLLMER. - The Journal of Pediatric. 16 mayo j940. Pág. 63.

VOLLMER and GOLDBERGER. - Bull. Sá View Hospical 4: 317, 1939.

VOLILMER, H. ZEEISSON, C. and RUBIN: H. J. - Pediatric. 15: 508-1939.

STEWARD: J, of Pediatic. Vol. 13: 510. May 1939.

AMERICAN REVIEW. - 30: 713. 1934

VOLLMER, H. - Am. Journal Dis. Chil. 56: $584-1935$.

ANZEN. - American J. Dis. Chil. Vol. 50: 104, 1935.

SAYAGO and NAPUT. - Prena médica argentina. Vol. 26, 881. Miyo 1939.

F. C. TUCCI y J. B. GOMEZ, - Estudio comparativo entre la tuberculina antigua de Koch, 12 exotuberculina de Finzi $y$ \& derivado proteico purificado de Szibert y Long. Jornadas Platenses de Pediatría 21. 22 y 23 de Sept. I 939.

PESA CERECEDA y JULIO MENEGHELLO. - Considir cione; abre a!gun.rs anamolías en las raccionzs tubercuínicas in ef n:ño, Rev. Chilena de Pediatría N.9 9. 1939. 